\title{
An undiagnosed pseudoaneurysm found during arterial catheterization in a Takayasu arteritis patient
}

\author{
Jeong Jin Min, Yoonjung Shon, Hyun Joo Kim, Deok Man Hong, and Yunseok Jeon \\ Department of Anesthesiology and Pain Medicine, Seoul National University Hospital, Seoul, Korea
}

Recently, the number of diagnostic and interventional angiographic procedures performed is increasing. Many patients who undergo surgery receive various angiographic procedures. Some of them may have complications related to the procedures. One of the complications, femoral pseudoaneurysm (PSA), is a complication that follows femoral arterial cannulation for angiographic procedures [1]. We would like to report a case of an undiagnosed right femoral artery PSA found during a right femoral arterial line insertion.

A 60-year old female patient, $161 \mathrm{~cm}$ in height and 51.7 $\mathrm{kg}$ in weight, was admitted for heart valve surgery. She had hypertension, severe mitral regurgitation, moderate aortic regurgitation and atrial fibrillation. She was taking antihypertensives and warfarin. During the preoperative evaluation, Takayasu arteritis was diagnosed. A preoperative CT angiogram showed bilateral total occlusion of the subclavian arteries and severe stenosis of the right and left common carotid arteries. Thus, the surgical plan was changed from heart valve surgery to innominate to left common carotid artery bypass surgery. Preoperative coronary angiography was performed via the right femoral artery three days before the operation. In the operating room, arterial cannulation of the right femoral artery was attempted. We tried to insert a femoral arterial line because the patient's bilateral brachial arterial stenosis made her radial artery unsuitable for an arterial line. Also, since an arterial line in the dorsalis pedis artery is easily underdamped, our first choice was the femoral artery. On visual inspection, there were no abnormalities of the right inguinal area except a small skin scar. An 18 gauge needle was inserted after palpation for the most pulsatile area. Blood was aspirated in the first attempt. However, the guide wire could not be advanced through the inserted needle. After several attempts, we used an ultrasoundguided technique to insert the wire and a PSA was found (Fig. 1). The PSA communicated with the right femoral artery through a small channel, and the guide wire was in the PSA. Therefore, we inserted an arterial line into the left dorsalis pedis artery. The resection of the PSA and the innominate to carotid bypass were done under general anesthesia and were uneventful.

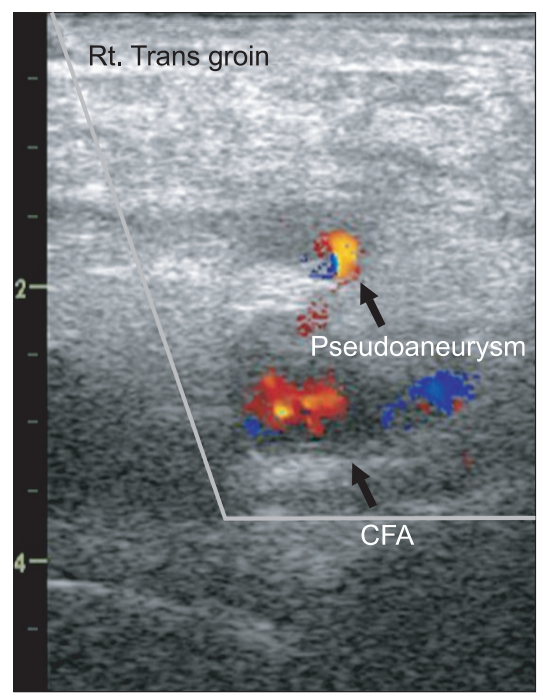

Fig. 1. Doppler image of the right femoral artery, showing a pseudoaneurysm communicating with the common femoral artery; CFA: common femoral artery.

Corresponding author: Yunseok Jeon, M.D., Ph.D., Department of Anesthesiology and Pain Medicine, Seoul National University Hospital, Yeongun-dong, Jongro-gu, Seoul 110-744, Korea. Tel: 82-2-2072-2465, Fax: 82-2-747-5639, E-mail: jeonyunseok@gmail.com

(c) This is an open-access article distributed under the terms of the Creative Commons Attribution Non-Commercial License (http:// creativecommons.org/licenses/by-nc/3.0/), which permits unrestricted non-commercial use, distribution, and reproduction in any medium, provided the original work is properly cited. 
A PSA is a hematoma which communicates with the arterial circulation but is not surrounded by an arterial wall. It is distinguished from a true aneurysm which is a localized dilatation of an artery [2]. The femoral PSA is a complication that follows femoral arterial cannulation for angiographic procedures. The risk factors for femoral PSA formation include procedural and patient factors [3]. Procedural factors are low femoral puncture into the superficial femoral or profunda femoris artery, arterial cannulation for interventional purposes rather than diagnostic purposes, and inadequate compression after the procedure. Patient factors are obesity, anticoagulation, hemodialysis and calcified arteries. In this case, the patient was not obese and the puncture site was at the common femoral artery but she was taking warfarin due to atrial fibrillation. Doppler ultrasound has been the main method of diagnosis of PSA [1]. Typical findings include swirling color flow seen in a mass separate from the affected artery, and color flow within a tract communicating with the mass and the affected artery as a PSA neck [4]. The treatment options of PSA include conservative management, ultrasound-guided compression, minimally invasive percutaneous therapies (thrombin or collagen injection and coil embolization), and surgical repair [2]. Percutaneous ultrasoundguided thrombin injection is currently the treatment of choice in many centers due to advantages such as a shorter procedural time, better patient tolerance and higher effectiveness in patients on anticoagulants, compared with ultrasound-guided compression [2]. Indications for surgical treatment are rapid expansion of the PSA, failure of percutaneous intervention, concomitant distal ischemia, and neurological deficits [1]. The PSA of this patient did not meet the indications for surgery. However, considering that the patient was already anesthetized, surgical repair as a definitive treatment was performed. Complications of PSA include rupture, distal embolization, local pain, neuropathy and local skin ischemia from its mass effect
[4]. According to the medical record, a vascular closure device (Perclose ProGlide $^{\mathrm{TM}}$, Abbott Vascular Inc., Redwood City, CA, USA) was used after the coronary angiography but the impact of arterial puncture closure devices on PSA formation is unclear [5].

The number of angiographic procedures being performed and the number of patients who need invasive blood pressure monitoring during surgery are constantly increasing. Many patients who need angiographic procedures before surgery also need invasive blood pressure monitoring. As in this case, options for sites for insertion of an arterial line may be limited because of underlying disease, vascular status, and the type of surgery involved. If the artery selected for cannulation was previously an access site for a percutaneous angiographic procedure, ultrasonographic examination of the artery before cannulation may be useful for avoiding further complications. Also, if an arterial line cannot be inserted even after repeated attempts, an anesthesiologist may consider examination of the puncture site with an ultrasound device.

\section{References}

1. Lenartova $\mathrm{M}$, Tak T. Iatrogenic pseudoaneurysm of femoral artery: case report and literature review. Clin Med Res 2003; 1: 243-7.

2. Ahmad F, Turner S, Torrie P, Gibson M. Iatrogenic femoral artery pseudoaneurysms--a review of current methods of diagnosis and treatment. Clinl Radiol 2008; 63: 1310-6.

3. Katzenschlager R, Ugurluoglu A, Ahmadi A, Hülsmann M, Koppensteiner $\mathrm{R}$, Larch E, et al. Incidence of pseudoaneurysm after diagnostic and therapeutic angiography. Radiology 1995; 195: 463-6.

4. Eisenberg L, Paulson EK, Kliewer MA, Hudson MP, DeLong DM, Carroll BA. Sonographically guided compression repair of pseudoaneurysms: further experience from a single institution. AJR Am J Roentgenol 1999; 173: 1567-73.

5. Vaitkus PT. A meta-analysis of percutaneous vascular closure devices after diagnostic catheterization and percutaneous coronary intervention. J Invasive Cardiol 2004; 16: 243-6. 